\title{
Airway inflammation in chronic obstructive pulmonary disease
}

\author{
Nikolaos Angelis', Konstantinos Porpodis', Paul Zarogoulidis', Dionysios Spyratos', loannis Kioumis', Antonis \\ Papaiwannou', Georgia Pitsiou', Kosmas Tsakiridis ${ }^{2}$, Andreas Mpakas ${ }^{2}$, Stamatis Arikas ${ }^{2}$, Theodora Tsiouda ${ }^{3}$, \\ Nikolaos Katsikogiannis ${ }^{4}$, loanna Kougioumtzi ${ }^{4}$, Nikolaos Machairiotis ${ }^{4}$, Michael Argyriou ${ }^{5}$, George Kessisis ${ }^{6}$, \\ Konstantinos Zarogoulidis' \\ 'Pulmonary Department-Oncology Unit, "G. Papanikolaou” General Hospital, Aristotle University of Thessaloniki, Thessaloniki, Greece; \\ ${ }^{2}$ Cardiology Department, "Saint Luke” Private Clinic, Thessaloniki, Panorama, Greece; ${ }^{3}$ Internal Medicine Department, "Theageneio" \\ Cancer Hospital, Thessaloniki, Greece; ${ }^{4}$ Surgery Department (NHS), University General Hospital of Alexandroupolis, Democritus \\ University of Thrace, Alexandroupolis, Greece; ${ }^{5}$ 2nd Cardiac Surgery Department, "Evangelismos” General Hospital, Athens, Greece; \\ ${ }^{6}$ Oncology Department, "Saint Luke” Private Clinic, Thessaloniki, Panorama, Greece
}

\begin{abstract}
Chronic obstructive pulmonary disease (COPD) is an inflammatory airway disease whose incidence and mortality increases every year. It is associated with an abnormal inflammatory response of the lung to toxic particles or gases (usually cigarette smoke). A central role in the pathophysiology has been shown to play a chronic inflammation of the airways that is expressed primarily by hypersecretion of mucus, stenosis of the smaller airways and the establishment of pulmonary emphysema. There is an increasing trend for assessing the inflammatory pattern of inflammatory airway diseases through mediators measured by noninvasive techniques. Markers in biological fluids and exhaled air have been the subject of intense evaluation over the past few years, with some of them reaching their introduction into clinical practice, while others remain as research tools. Of particular interest for the scientific community is the discovery of clinically exploitable biomarkers associated with specific phenotypes of the disease. Studying the effects of therapeutic interventions in these biomarkers may lead to targeted therapy based on phenotype and this is perhaps the future of therapeutics in COPD.

KEYWORDS Chronic obstructive pulmonary disease (COPD); pathophysiology; airway inflammation; biomarkers; non-invasive techniques
\end{abstract}

J Thorac Dis 20I4;6(SI):SI67-SI72. doi: I0.3978/j.issn.2072-I439.20/4.03.07

Chronic obstructive pulmonary disease (COPD) is a common respiratory condition involving the airways and characterized by airflow limitation $(1,2)$. It affects more than 5 percent of the population and is associated with high morbidity and mortality (3). It is the third-ranked cause of death in the United States, killing more than 120,000 individuals each year (4). COPD is the only one of the six leading causes of death in the U.S. whose

Correspondence to: Paul Zarogoulidis. Pulmonary Department, “G. Papanikolaou” General Hospital, Aristotle University of Thessaloniki, Thessaloniki, Greece. Email: pzarog@hotmail.com.

Submitted Mar 02, 2014. Accepted for publication Mar 04, 2014.

Available at www.jthoracdis.com

ISSN: 2072-1439

(C) Pioneer Bioscience Publishing Company. All rights reserved. mortality is increasing. It is estimated that 2020 will be the third leading cause of death worldwide after ischemic heart disease and cerebrovascular disease $(5,6)$. As a consequence of its high prevalence and chronicity, it causes high resource utilization with frequent clinician office visits, frequent hospitalizations due to acute exacerbations, and the need for chronic therapy (e.g., supplemental oxygen therapy, medication) (1).

Correct diagnosis of COPD is important because appropriate management can decrease symptoms (especially dyspnea), reduce the frequency and severity of exacerbations, improve health status, improve exercise capacity, and prolong survival (7). The Global Initiative for Chronic Obstructive Lung Disease (GOLD) defines COPD as follows: COPD, a common preventable and treatable disease, is characterized by airflow limitation that is usually progressive and associated with an enhanced chronic inflammatory response in the airways and the lung to noxious 
particles or gases. Exacerbations and comorbidities contribute to the overall severity in individual patients (8).

Central role in the pathophysiology of COPD has been shown to play a chronic inflammation of the airways that is expressed primarily by increased numbers of goblet cells, mucus gland hyperplasia, fibrosis, narrowing and reduction in the number of small airways and airway collapse due to the loss of tethering caused by alveolar wall destruction in emphysema (9). Pulmonary emphysema is associated with increased activity of proteolytic enzymes (MMP-2, MMP-9, MMP-12, cathepsin $\mathrm{K}, \mathrm{L}$ and $\mathrm{S}$ and neutrophil elastase) which are activated due to inflammation and oxidative stress occurring in COPD. Mucus hyper secretion, which can be associated with an increased number of exacerbations, possibly radiate inflammatory lesion around the submucosal glands and severity of inflammation in most peripheral airways $(10,11)$.

Macrophages play a key role in inflammation of COPD. Smoking and other inhaled irritants cause inflammatory response in the peripheral airways and lung parenchyma. The activated macrophages release of inflammation mediators and chemotactic factors, including tumor necrosis factor-a (TNF-a), interleukin IL-6, interleukin IL-8, monocyte chemotactic peptide (MCP)-1, leukotriene LTB4 and reactive oxygen species, and secrete proteolytic enzymes (especially MMP-9, MMP-12) whose action contributes to the establishment of pulmonary emphysema. Under the influence of secreted chemotactic factors (particularly IL-8 and LTB-4), neutrophils move directly to the respiratory tract and cause the over-stimulation of submucosal mucous glands and goblet cells by proteinases (especially neutrophil elastase, cathepsin-G, proteinase-3) secreted from these (12-16).

Bronchial biopsies from COPD patients also showed infiltration of the bronchi by CD4+ and CD8+ predominantly $\mathrm{T}$ lymphocytes (17-22). There is a correlation between the number of T-lymphocytes, the extent of alveolar destruction and the rate of airway obstruction (12). The role of CD8+ lymphocytes is not fully understood. It is believed that CD8+ lymphocytes have the ability of lysis and apoptosis of alveolar epithelial cells through the secretion of perforins, granzyme-B and TNF-a. There is a correlation of the CD8+ lymphocytes with increased apoptosis of alveolar cells in emphysema (23).

Remarkable role in COPD inflammation are playing dendritic and epithelial cells. Dendritic cells play a central role in initiating immune response activating a variety of inflammatory and immune cells including macrophages, B and T-lymphocytes and neutrophils (24). They form a rich network into airways and lung parenchyma, which is located near the surface, so that it can distinguish inhaled toxic substances $(25,26)$. The epithelial cells of the airways and alveoli are a major source of inflammatory mediators and proteases. Triggered by smoking, they secrete various factors such as TNF-a, the TGF-b, interleukins IL-1b and IL-8 and GM-CSF (granulocyte-macrophage colony stimulating factor), which cause activation of fibroblasts and small airways fibrosis (27-29). The epithelial cells contribute to the defense of the body through the production of mucus which traps bacteria and through substances, such as defensins and cationic proteins, which have antimicrobial properties $(30,31)$. The effect of harmful factors on their surface results in their squamous metaplasia, disruption of their action and the submucosal gland and goblet cell metaplasia (13).

Finally, dominant position in the pathophysiology of COPD holds oxidative stress which is installed when the production of active oxygen radicals overcomes the antioxidant defense mechanisms of the body. This results in serious damage to lipids, proteins and cell DNA (disturbed protease-antiprotease balance) (32-34). The reactive oxygen species are produced by cells such as neutrophils, macrophages, eosinophils and epithelial cells, when activated by the inflammation in the respiratory tract (33). Main indicators of oxidative stress in the body are the hydrogen peroxide $\left(\mathrm{H}_{2} \mathrm{O}_{2}\right)$ and 8-isoprostaglandin $\mathrm{F} 2 \mathrm{a}$ (8-isoprostane) which is elevated in exhaled air (35-39).

Assessment of airway inflammation was performed until recently only with invasive techniques such as the bronchoalveolar lavage (BAL), or intrabronchial and transbronchial biopsy. However, such techniques are not always feasible to use nor are measures of daily clinical practice (40). The need for noninvasive assessment of airway inflammation is imperative, since inflammatory airway diseases, are usually characterized by variation in their clinical presentation throughout their course. Moreover, there is an increasing trend for assessing the inflammatory pattern of inflammatory airway diseases through mediators measured by noninvasive techniques (41). Markers in biological fluids and exhaled air have been the subject of intense evaluation over the past few years, with some of them reaching their introduction into clinical practice, while others remain as research tools (42). The most studied and used non-invasive techniques are induced sputum, biomarkers measurement in exhaled air [mainly represented by exhaled nitric oxide (NO)] and exhaled breath condensate (EBC) (40).

Induced sputum is a noninvasive technique that enables clinical researchers to elucidate the course of several inflammatory airway infections, including COPD. Its main advantage lies in making, processing and analysis fully thought out methodology. Inhalation of isotonic or hypertonic solutions administered by nebulisation has been demonstrated to induce a small amount of airway secretion that can be expectorated and analyzed. The 
Table 1. Inflammatory process of chronic obstructive pulmonary disease (COPD).

\begin{tabular}{|c|c|c|c|}
\hline Inflammation cells & Stimulators & Inflammatory mediators & $\begin{array}{l}\text { Increased inflammatory } \\
\text { mediators }\end{array}$ \\
\hline Macrophages & $\begin{array}{l}\text { Inhaled irritants, dendritic } \\
\text { cells }\end{array}$ & $\begin{array}{l}\text { TNF-a, IL-6, IL-8, MCP-I, LTB4 and reactive oxygen } \\
\text { species, MMP-9, MMPI } 2\end{array}$ & $\begin{array}{l}\text { Oxidative stress } \\
\uparrow \mathrm{H}_{2} \mathrm{O}_{2}\end{array}$ \\
\hline $\begin{array}{l}\text { CD8+ T } \\
\text { lymphocytes }\end{array}$ & $\begin{array}{l}\text { Inhaled irritants, dendritic } \\
\text { cells }\end{array}$ & Perforins, grazyme-B, TNF-a & $\uparrow 8$-isoprostane \\
\hline Dendritic cells & Inhaled irritants & Macrophages, B and T-lymphocytes and neutrophils & \\
\hline Epithelial cells & Inhaled irritants & TNF-a, the TGF-b, interleukins IL-Ib and IL-8 and GM-CSF & \\
\hline Neutrophils & Macrophages, dendritic cells & Neutrophil elastase, cathepsin-G, proteinase- 3 & \\
\hline
\end{tabular}

mechanisms by which this occurs are not known, but both direct and indirect mechanisms are likely to be involved. It is believed that the increased osmolarity of the airway lining fluid increases vascular permeability in bronchial mucosa and induces production of mucus by submucosal glands (43). Induced sputum is a reproducible method, sensitive and reliable whose value is not limited to detection of sputum inflammatory mediators. The main clinical use is associated with the recognition of eosinophilic inflammation and the utilization of eosinophils as a tool for guiding treatment and monitoring the course of patients with asthma and COPD $(44,45)$.

The main and most important representative of the measured biomarkers in exhaled air is the fraction of exhaled nitric oxide (FeNO), which is the most studied biomarker today. $\mathrm{NO}$ in orally exhaled air mainly originates from the respiratory epithelium. $\mathrm{NO}$ is produced by inducible $\mathrm{NO}$ synthase (iNOS), which is regulated by signal transducer and activator of transcription (STAT)-1 under the influence of homeostatic interferon-c (46). The main limitation to the use of FeNO lies in the fact that smoking has a negative effect on its concentration. The main application is in asthma which provides fast information on eosinophilic inflammation in the airways of asthmatic individuals. It was also recognized early on that FeNO is increased in at least some subgroups of patients with COPD $(47,48)$. It was later suggested that this increase correlated with bronchial reversibility to salbutamol and thus could represent an asthma-like component of COPD (49). In recent years is investigated the potential role of FeNO in predicting response to treatment in acute exacerbations of COPD, and the likelihood that FeNO be an indicator of treatment response to corticosteroids in patients with COPD (50-53).

The assessment of markers of airway inflammation/oxidative stress in EBC has been suggested as a simple, rapid, safe and noninvasive technique that is suitable for assessment of airway inflammation in humans of all ages $(41,54-56)$. EBC is formed by cooling of exhaled breath of patients in a condenser system $(54,55)$. Whereas volatile mediators of airway inflammation/ oxidative stress are mainly recovered in the gas phase of exhaled breath, nonvolatile biomarkers from the epithelial lining fluid of the airway epithelium are released in aerosol particles, which can be measured in EBC $(54,55)$. Several markers in EBC can be assessed simultaneously. Potentially, noninvasive biomarkers in EBC may be of help in the discrimination between different inflammatory phenotypes, the assessment of the severity of airway inflammation, to monitor treatment and disease control, and to predict disease exacerbations at an early stage $(41,54,56)$. Besides these potential "pearls" of EBC measurements, there are a number of pitfalls: (I) the concentration of markers is low, thus ultrasensitive techniques are required for analysis; (II) many factors may have an influence on biomarkers in EBC (Table 1); and (III) there is variation in results between different centers. Molecules that are measured in $\mathrm{EBC}$ are $\mathrm{H}_{2} \mathrm{O}_{2}$, derivatives of $\mathrm{NO}$, prostanoids (prostaglandins and thromboxanes), leukotrienes, 8-isoprostane, EBC ph and other biomarkers such as cytokines (interleukins and TNF-a) and various proteins $(56,57)$.

The main therapeutic option for the treatment of chronic inflammation of asthmatic patients is corticosteroids. However, despite proven inflammatory background of COPD, corticosteroids do not seem to have any effect on disease's inflammation. No changes in neutrophilic inflammation and no decrease in inflammatory mediators and proteases are observed after their use. There is an opposite effect of corticosteroids in granulocytes with decreased survival of eosinophils and prolongation of life of the neutrophils. This has led to the failure of treatment to stop the course of COPD and demonstrates the need for discovery of new therapeutic interventions (13).

The need for noninvasive evaluation of airway inflammation and the discovery of targeted therapeutic interventions is imperative since COPD is characterized by variable clinical manifestations during the course of and resistance to standard 
anti-inflammatory therapy. The biomarkers in biological fluids and exhaled air were the subject of intense study in previous years and others than those used today in clinical practice and others are still under investigation. The central question is whether the information obtained from measurements of inflammatory mediators currently fulfills the conditions required to characterize them as appropriate biomarkers. An imaginary biomarker should be evaluated by a standard protocol, must have an acceptable reproducibility, should demonstrate the specificity of the disease and must have the ability to detect either changes attributable to therapeutic interventions or changes in the health status (e.g., an exacerbation of the disease). With the techniques available today is difficult to detect imaginary biomarker that meets the above conditions (58).

COPD is a heterogeneous entity that includes a variety of obstructive diseases that differ considerably on their mechanisms of action and response to treatment. Hence the need to recognize the different phenotypes of the disease within the range of these syndromes is vital for patient management. The ideal approach is not measuring the levels of a particular biomarker, but the effort to recognize the specific phenotype associated with specific biomarker and underlying pathophysiology. The combination, in fact, of different biomarkers can approach more effectively the classification of a particular phenotype. The knowledge of the pathophysiology of epidemic diseases, such as COPD, is essential for the management of patients and to evaluate the effects of new treatments for them and non-invasive diagnostic techniques help in this direction. Based on the current literature shows that combining different non-invasive diagnostic methods by which samples are taken from different compartments of the airways is possible to assess in detail the different mediators of inflammation and to monitor the effectiveness of treatment. In the future, the exploitation of this specialized monitoring of inflammation may allow each phenotype of the disease to have a characteristic profile of exhaled biomarkers and studying the effects of therapeutic interventions in these biomarkers may lead to targeted therapy based on phenotype and this is perhaps the future of therapeutics in COPD (58).

\section{Acknowledgements}

Disclosure: The authors declare no conflict of interest.

\section{References}

1 Buist AS, McBurnie MA, Vollmer WM, et al. International variation in the prevalence of COPD (the BOLD Study): a population-based prevalence study. Lancet 2007;370:741.
2 Gershon AS, Warner L, Cascagnette P, et al. Lifetime risk of developing chronic obstructive pulmonary disease: a longitudinal population study. Lancet 2011;378:991-6.

3 Centers for Disease Control and Prevention (CDC). Chronic obstructive pulmonary disease among adults--United States, 2011. MMWR Morb Mortal Wkly Rep 2012;61:938-43.

4 Miniño AM, Murphy SL, Xu J, et al. Deaths: final data for 2008. Natl Vital Stat Rep 2011;59:1-126.

5 Murray CJ, Lopez AD. Alternative projections of mortality and disability by cause 1990-2020: Global Burden of Disease Study. Lancet 1997;349:1498-504.

6 Mannino DM, Homa DM, Akinbami LJ, et al. Chronic obstructive pulmonary disease surveillance--United States, 1971-2000. MMWR Surveill Summ 2002;51:1-16.

7 Rennard SI, Vestbo J. COPD: the dangerous underestimate of 15\%. Lancet 2006;367:1216-9.

8 Global strategy for the diagnosis, management, and prevention of chronic obstructive pulmonary disease: Revised 2014. Global Initiative for Chronic Obstructive Lung Disease (GOLD). Available online: www.goldcopd.org (Accessed on January 23, 2014).

9 McDonough JE, Yuan R, Suzuki M, et al. Small-airway obstruction and emphysema in chronic obstructive pulmonary disease. N Engl J Med 2011;365:1567-75.

10 Kanner RE, Anthonisen NR, Connett JE. Lower respiratory illnesses promote FEV1 decline in current smokers but not ex-smokers with mild chronic obstructive pulmonary disease: results from the lung health study. Am J Respir Crit Care Med 2001;164:358-64.

11 Saetta M, Turato G, Facchini FM, et al. Inflammatory cells in the bronchial glands of smokers with chronic bronchitis. Am J Respir Crit Care Med 1997;156:1633-9.

12 Barnes PJ, Shapiro SD, Pauwels RA. Chronic obstructive pulmonary disease: molecular and cellular mechanisms. Eur Respir J 2003;22:672-88.

13 Barnes PJ. Mechanisms in COPD: differences from asthma. Chest 2000;117:10S-4S.

14 Bucchioni E, Kharitonov SA, Allegra L, et al. High levels of interleukin-6 in the exhaled breath condensate of patients with COPD. Respir Med 2003;97:1299-302.

15 Sommerhoff CP, Nadel JA, Basbaum CB, et al. Neutrophil elastase and cathepsin $\mathrm{G}$ stimulate secretion from cultured bovine airway gland serous cells. J Clin Invest 1990;85:682-9.

16 Witko-Sarsat V, Halbwachs-Mecarelli L, Schuster A, et al. Proteinase 3, a potent secretagogue in airways, is present in cystic fibrosis sputum. Am J Respir Cell Mol Biol 1999;20:729-36.

17 Retamales I, Elliott WM, Meshi B, et al. Amplification of inflammation in emphysema and its association with latent adenoviral infection. Am J Respir Crit Care Med 2001;164:469-73.

18 Finkelstein R, Fraser RS, Ghezzo H, et al. Alveolar inflammation and its relation to emphysema in smokers. Am J Respir Crit Care Med 1995; 152:1666-72. 
19 Majo J, Ghezzo H, Cosio MG. Lymphocyte population and apoptosis in the lungs of smokers and their relation to emphysema. Eur Respir J 2001;17:946-53.

20 Saetta M, Baraldo S, Corbino L, et al. CD8+ cells in the lungs of smokers with chronic obstructive pulmonary disease. Am J Respir Crit Care Med 1999;160:711-7.

21 O'Shaughnessy TC, Ansari TW, Barnes NC, et al. Inflammation in bronchial biopsies of subjects with chronic bronchitis: inverse relationship of CD8+ T lymphocytes with FEV1. Am J Respir Crit Care Med 1997; 155:852-7.

22 Saetta M, Di Stefano A, Turato G, et al. CD8+ T-lymphocytes in peripheral airways of smokers with chronic obstructive pulmonary disease. Am J Respir Crit Care Med 1998;157:822-6.

23 Hashimoto S, Kobayashi A, Kooguchi K, et al. Upregulation of two death pathways of perforin/granzyme and FasL/Fas in septic acute respiratory distress syndrome. Am J Respir Crit Care Med 2000;161:237-43.

24 Banchereau J, Briere F, Caux C, et al. Immunobiology of dendritic cells. Annu Rev Immunol 2000;18:767-811.

25 Holt PG, Stumbles PA. Regulation of immunologic homeostasis in peripheral tissues by dendritic cells: the respiratory tract as a paradigm. J Allergy Clin Immunol 2000;105:421-9.

26 Huang Q, Liu D, Majewski P, et al. The plasticity of dendritic cell responses to pathogens and their components. Science 2001;294:870-5.

27 Mio T, Romberger DJ, Thompson AB, et al. Cigarette smoke induces interleukin-8 release from human bronchial epithelial cells. Am J Respir Crit Care Med 1997; 155:1770-6.

28 Hellermann GR, Nagy SB, Kong X, et al. Mechanism of cigarette smoke condensate-induced acute inflammatory response in human bronchial epithelial cells. Respir Res 2002;3:22.

29 Floreani AA, Wyatt TA, Stoner J, et al. Smoke and C5a induce airway epithelial ICAM-1 and cell adhesion. Am J Respir Cell Mol Biol 2003;29:472-82.

30 Adler KB, Li Y. Airway epithelium and mucus: intracellular signaling pathways for gene expression and secretion. Am J Respir Cell Mol Biol 2001;25:397-400.

31 Aarbiou J, Rabe KF, Hiemstra PS. Role of defensins in inflammatory lung disease. Ann Med 2002;34:96-101.

32 Repine JE, Bast A, Lankhorst I. Oxidative stress in chronic obstructive pulmonary disease. Am J Respir Crit Care Med 1997;156:341-57.

33 MacNee W. Oxidative stress and lung inflammation in airways disease. Eur J Pharmacol 2001;429:195-207.

34 Henricks PA, Nijkamp FP. Reactive oxygen species as mediators in asthma. Pulm Pharmacol Ther 2001;14:409-20.

35 Kharitonov SA, Barnes PJ. Exhaled markers of pulmonary disease. Am J Respir Crit Care Med 2001;163:1693-722.

36 Montuschi P, Barnes PJ. Analysis of exhaled breath condensate for monitoring airway inflammation. Trends Pharmacol Sci 2002;23:232-7.

37 Paredi P, Kharitonov SA, Barnes PJ. Analysis of expired air for oxidation products. Am J Respir Crit Care Med 2002;166:S31-7.

38 Montuschi P, Collins JV, Ciabattoni G, et al. Exhaled 8-isoprostane as an in vivo biomarker of lung oxidative stress in patients with COPD and healthy smokers. Am J Respir Crit Care Med 2000;162:1175-7.

39 Biernacki WA, Kharitonov SA, Barnes PJ. Increased leukotriene B4 and 8 -isoprostane in exhaled breath condensate of patients with exacerbations of COPD. Thorax 2003;58:294-8.

40 O’Byrne PM, Hargreave FE. Non-invasive monitoring of airway inflammation. Am J Respir Crit Care Med 1994;150:S100-2.

41 Kharitonov SA, Barnes PJ. Exhaled biomarkers. Chest 2006;130:1541-6.

42 Taylor DR, Pavord ID. Biomarkers in the assessment and management of airways diseases. Postgrad Med J 2008;84:628-34.

43 Umeno E, McDonald DM, Nadel JA. Hypertonic saline increases vascular permeability in the rat trachea by producing neurogenic inflammation. J Clin Invest 1990;85:1905-8.

44 Green RH, Brightling CE, McKenna S, et al. Asthma exacerbations and sputum eosinophil counts: a randomised controlled trial. Lancet 2002;360:1715-21.

45 Siva R, Green RH, Brightling CE, et al. Eosinophilic airway inflammation and exacerbations of COPD: a randomised controlled trial. Eur Respir J 2007;29:906-13.

46 Alving K, Malinovschi A. Basic aspects of exhaled nitric oxide. Eur Respir Mon 2010;49:1-31.

47 Maziak W, Loukides S, Culpitt S, et al. Exhaled nitric oxide in chronic obstructive pulmonary disease. Am J Respir Crit Care Med 1998; 157:998-1002.

48 Corradi M, Majori M, Cacciani GC, et al. Increased exhaled nitric oxide in patients with stable chronic obstructive pulmonary disease. Thorax 1999;54:572-5.

49 Papi A, Romagnoli M, Baraldo S, et al. Partial reversibility of airflow limitation and increased exhaled NO and sputum eosinophilia in chronic obstructive pulmonary disease. Am J Respir Crit Care Med 2000;162:1773-7.

50 Taylor DR, Pavord ID. Biomarkers in the assessment and management of airways diseases. Postgrad Med J 2008;84:628-34; quiz 633.

51 Smith AD, Cowan JO, Brassett KP, et al. Use of exhaled nitric oxide measurements to guide treatment in chronic asthma. N Engl J Med 2005;352:2163-73.

52 Shaw DE, Berry MA, Thomas M, et al. The use of exhaled nitric oxide to guide asthma management: a randomized controlled trial. Am J Respir Crit Care Med 2007; 176:231-7.

53 Barnes PJ, Dweik RA, Gelb AF, et al. Exhaled nitric oxide in pulmonary diseases: a comprehensive review. Chest 2010;138:682-92.

54 Rosias PP, Dompeling E, Hendriks HJ, et al. Exhaled breath condensate in children: pearls and pitfalls. Pediatr Allergy Immunol 2004;15:4-19.

55 Horváth I, Hunt J, Barnes PJ, et al. Exhaled breath condensate: methodological recommendations and unresolved questions. Eur Respir J 2005;26:523-48.

56 Hunt J. Exhaled breath condensate: an evolving tool for noninvasive 
evaluation of lung disease. J Allergy Clin Immunol 2002;110:28-34.

57 Koutsokera A, Loukides S, Gourgoulianis KI, et al. Biomarkers in the exhaled breath condensate of healthy adults: mapping the path towards reference values. Curr Med Chem 2008;15:620-30.

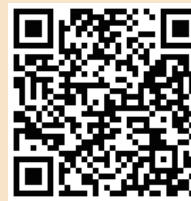

Cite this article as: Angelis N, Porpodis K, Zarogoulidis P, Spyratos D, Kioumis I, Papaiwannou A, Pitsiou G, Tsakiridis K, Mpakas A, Arikas S, Tsiouda T, Katsikogiannis N, Kougioumtzi I, Machairiotis N, Argyriou M, Kessisis G, Zarogoulidis K. Airway inflammation in chronic obstructive pulmonary disease. J Thorac Dis 2014;6(S1):S167-S172. doi: 10.3978/ j.issn.2072-1439.2014.03.07
Loukides S, Kostikas K, Barnes PJ. eds. Non-invasive assessment of airways inflammation in Asthma and COPD. Athens: Paschalidis Medical Publications, 2011. 